\title{
CONSTRUCTING DIGITAL ARTIFACTS ON THE WEB
}

\author{
Manasa M.B ${ }^{1}$, Gousia Thaniyath ${ }^{2}$ \\ ${ }^{1}$ Computer Science and Engineering, The Oxford college of Engineering, and Bangalore \\ manasalavakumar@gmail.com \\ ${ }^{2}$ Computer Science and Engineering, The Oxford college of Engineering, and Bangalore \\ gous.tans@gmail.com
}

\begin{abstract}
The present digital technology innovation happens by replicating the existing distributed Web assets and extending to incorporate new features. It's a chain of events happened over and over on the web which leads to an act of sharing or publishing digital content on the web. These web assets incorporates digital type of datasets, code, messages, Process and Media however there is no formal way of sharing instruments took after to make advanced curios on the Web. Which are provable, one of a kind and unique. These deficiencies have a genuine negative effect on the capacity to replicate the outcomes, which in turn vigorously affects technology where reproducibility is vital. To take care of this issue, this paper presents believable Universal Resource Indicators contains encrypted notations which demonstrates how believable Universal Resource Indicatorsutilized to check the digital content. We exhibit how the substance of these records get to be one of a kind, including conditions to outer computerized artifacts and in this way the importance of provability for whole reference chain. Assessment of this reference executions demonstrates that all these outline objectives are accomplished by our methodology including huge documents. Our implementation holds good for the all levels in the Web, for example, transparent and fragmented engineering, which is completely good for present conventional models.
\end{abstract}

Keywords: Data Mining, Digital Technology, Nano Publications. - $* * *$

\section{INTRODUCTION}

Now a day's technology growth is huge specifically in digital technology, recreation is critical. Provable, unique, and originality are a vital fixing for making the results of mechanized procedures replicable, be that as it may, the present Web offers no ordinarily acknowledged strategies to guarantee these properties. Triesfor example, the Web to distribute information in a digitized way shows the issue, in which digital calculations working on hugemeasures of information can be relied upon to be much more original than people to be controlled or manipulated substance. Without suitable counter-measures, unidentified attackers can harm or trap such calculations by including only a few deliberately controlled things to extensive arrangements of data information. To take care of this issue, we propose a way to deal with make things on the (Semantic) Web certain, unique, what's more, original. This methodology for Uniform Resource Identifiers (URIs) contains cryptographic hash values and holds fast to the standards of the Web, in particular openness furthermore, decentralized design. Proposed system is an implementation and feature work of paper [1].

This methodology for Uniform Resource Identifiers (URIs) contains encrypted notationsand sticks to the standards of the Web, in particular transparent and fragmented design.Present paper we developed and updated form of a technical paper Anencrypted notations(once in a while called cryptographic review) are short arbitrary having succession of bytes (or, bits) which are ascertained way from an advanced artifacts[2], for example, a document. The same information dependably prompts the very same hash esteem, while only a negligibly altered data gives back a totally diverse quality. While there is an endlessness of conceivable inputs that prompt a particular given hash esteem, it is unthinkable practically speaking to remake any of the conceivable inputs just from the hash esteem. Present approach make a difference to a particular and permanent advanced artifacts.

\section{BACKGROUND}

An easy way of publishing digital content on the web, for example Nano distribution, which is nothing but sharing or publishing.

\section{A. Scientific Publishing}

Nanopublications can refer to different Nanopublications by means of their URIs, in this manner making complex scientific reference systems. Distributed Nanopublications should be unique, yet there is as of now no component to implement this[3]. It is surely understood that even artifactsthat should be Uniquewill be modified after some time for the same URI.

\section{B. Range of provability}

Nano distribution has range of provability based on the digital content. Suppose for a Nanopublication P1 that refers to another Nanopublication P2. In the event that you need to locate the substance of P2, you can basically hunt down it on the Web, not stressing whether the source is reliable or 
not, you just need to check whether the hash esteem really coordinates the substance.

\section{Requirements}

To take into account check of a given computerized artifactsas well as its whole reference tree. To take into consideration the incorporation of meta-information. The confirmation shouldbe on a theoretical basis but not on the bytes of a record, for various substance. It has to be conceivable for checking a computerized ancient rarity regardless of the fact that it is displayed in an alternate arrangement. Thismethodology has tobe fragmented and transparent. The methodology has tobe founded on current built up gauges and be good with current instruments and arrangements, so it can be utilized immediately.

\section{Properties}

Trusty URI artifactsare evident as in a recovered ancient rarity of the substance the URI is having. It specifically takes after permanent believable URI artifacts, many adjustment to existing substance additionally modifies its URI, in this manner making it another artifact. Once more, you can obviously change your artifactwhich is dependably similar to this. These are permanent on the web until somebody literally change the content of the artifact. In this circumstance, the artifact is not permanent because content has been modified. The trusty URI ensures that it is the ancient rarity you are searching for, regardless of the fact that the area of the stored artifact is not reliable or it was reserved from a dishonest source. Even though artifact is attacked but if content is not changed then it's truly permeant in nature. It gives complete acceptance of the artifacts in question for replicating the distributed innovation on the web.

\section{EXISTING SYSTEM}

There are various related methodologies in light of cryptographic hash values yet for the most part two methodologies are clarified underneath with the innovation utilized likewise with its points of interest and impediments. Web content corrupted by human beings and in existing, no methods to make web content unique.

\section{E. Git Version Control System.}

It utilizes hash qualities to distinguish submits of appropriated vaults. Profoundly Distributed archive submits can happen non-concurrently and anyplace even the separate site is disconnected from the net. Git don't characterize how advanced relics can be spoken to at a more theoretical level than their succession of bytes. Hash speaks to the byte substance of records. Git utilizes SHA-1 calculation, which is no more considered as secured. Self-references are not bolstered.

\section{F. Named Information URI's (ni URI's).}

It presents another URI convention ni, to implement advanced artifacts with hash values uniformly [4]. This methodology utilizes hash calculation, for example, SHA-
256 which is viewed as secure. Discretionary detail of a power, for example, example.org, where the ancient rarities can be found. Git don't characterize how advanced relics can be spoken to at a more unique level than their arrangement of bytes. ni-URI's don't bolster Self-references. Current programs don't perceives the ni-convention.

\section{PROPOSED SYSTEM}

The methodology displayed here is a perfect complimentary. Current believable URI's are more adaptable and give extra elements. Our main objective of creating experimental results more accurate by utilizing our methodology as a part of differing areas like bio-informatics[5], software engineering[6] and brain science[7]. Research articles [8] are proposed to package articlesfrom their dataset, coding languages, work processes. Our effective believableURI could be utilized to make such packages and different sorts of advanced artifacts we provable, unique and to implement their unchanging character such as permanent.

\section{IMPLEMENTATION}

We present a secluded methodology, which has got diverse system operates various types of operations on various calculated levels of abstraction, from byte level to abnormal state Formalisms. Other than that, the most intriguing elements of our methodology are self-references. The constructed believable URIs containsencrypted notations (a particular alphanumeric encoding plan) went before by a module identifier.This is an example:

http://localhost:8080/Lk5AbXdpz5DcaYXCh913eI9ruBosiL 5XDU3rxBbBaUG69

Everything that comes after 8080/is the part that is particular to trusty URIs, which we call artifacts code. In our methodology involves a specific movement of power: Once a trusty URI is built up, its artifacts code characterizes what object it confirms to, and the issuing power has no more the ability to change its significance.

\section{G. Modules}

Proposed system has been implemented using modules such as Data Owner, Web Server, Data Consumer and Attacker, as shown in Fig. 1.

In the data owner module, the authorpublish the data file. The content owner encrypts the content then stores on the Web server. The Data owner will be having all access rights for modifications for the encrypted data file. This content owner will send Meta data to Audit Web. In audit Web raw or metadata information is available for auditing and data integrity checking purpose. Data owner will create an end user with the access permission (read or write) to user. 


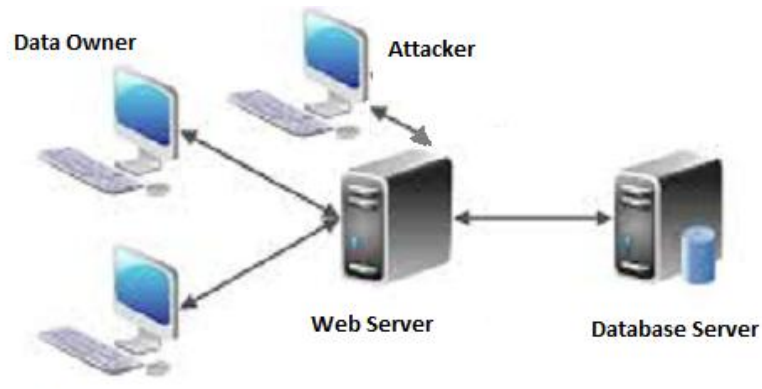

End USer

Fig. 1 System Architecture Diagram

The author can also audit the data integrity in the corresponding Web for verifying whether the data is safe or not using digital sign and web URL. If the data is not safe then he will delete the data and re upload the data to the corresponding Web server.

The Web server is responsible for data storage and file authorization for an end user. The data file will be stored with their tags such as file name, secret key, digital sign, and owner name. The data file will be sending based on the authentication. If the authenticationis correct then the data will be sent to the corresponding user and also will check the file name, consumer name and encrypted key. If all are true then it will send to the corresponding user or he will be captured as attacker. The Web server can also act as attacker to modify the data which will be auditing by the audit Web.

The data consumer is nothing but the end user who will request and gets file contents response from the corresponding Web servers. If the file name and encryptedkey, authentication is correct then the end is getting the file response from the Web or else he will be considered as an attacker and also he will be blocked in corresponding Web. If he wants to get the file after blocking he wants to remove from the Web.

Attacker is one who is integrating the Web file by adding malicious data to the corresponding Web. They may be within a Web or from outside the Web. If attacker is from inside the Web then those attackers are called as internal attackers. If the attacker is from outside the Web then those attackers are called as external attackers.

The trusty URI highlights gave by the exhibited libraries are additionally made accessible through an acceptance interface for Nano productions. Fig. 2 shows a system which offers truth be told significantly more than just acceptance. Clients can stack Nano productions in various ways, including recovery from URLs, afterward trusty URIs can be produced for them specifically by means of the Web interface. Verification of artifacts with trusty URIs is shown in Fig. 3.Nano distributions that as of now have a trusty URI are naturally confirmed and clients are educated about whether the confirmation was effective or not.
Owner Data Upload

\begin{tabular}{|c|c|c|}
\hline Select File & \multicolumn{2}{|l|}{ Choose file HTMLSourceCode.html } \\
\hline File Name & HTML & \\
\hline & 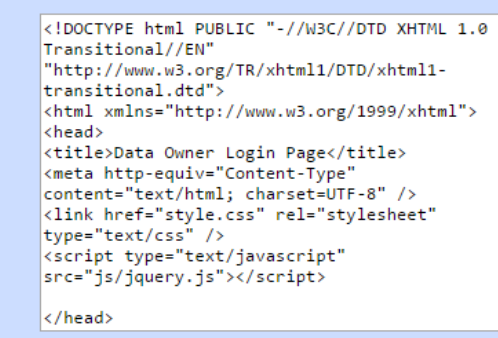 & 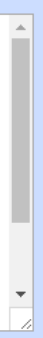 \\
\hline Digital Artifacts & $-5 c 0 e c 1339625 d d a 478 d c 23176807 f 36 f 120 d 9 a 93$ & \\
\hline & Encrypt & \\
\hline
\end{tabular}

Fig. 2 Generating Trusty URIs for Artifacts.

\section{EVALUATION RESULTS}

We performed some analyses on the trusty URI idea and its executions, in light of various arrangement of records. Verified the believable URI for every document of all usage that backing the particular arrangement. As shown in right sections of the Table 1 demonstrate resulted outcomes. All the legitimate records usage effectively confirmed their believable URIs. One byte modified artifacts has adifferent notations than the one of the believable URI.

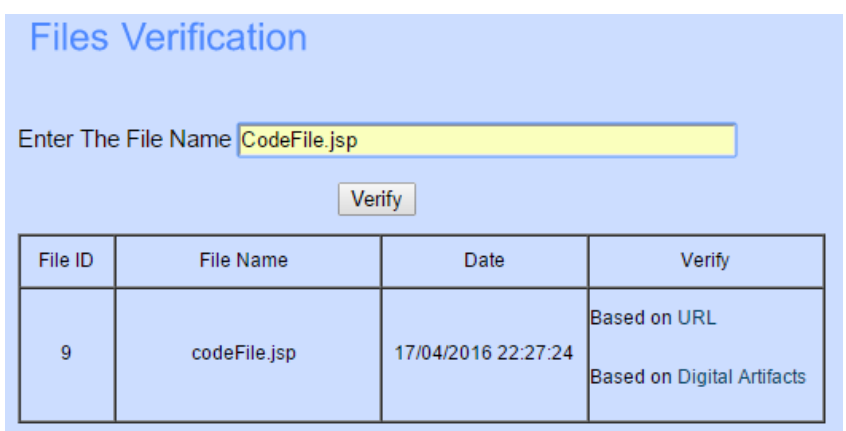

Fig. 3 Verification of Artifacts with trusty URIs

Table I. Analyses On The Trusty URI Idea And Its Executions Result

\begin{tabular}{|c|c|c|c|}
\hline \multirow{2}{*}{$\begin{array}{c}\text { Files } \\
\text { Format }\end{array}$} & \multicolumn{3}{|c|}{ Trusty URI Verification } \\
\cline { 2 - 4 } & valid & invalid & result \\
\hline Java JSP & $100 \%$ & $0 \%$ & Valid File \\
\hline Web HTML & $100 \%$ & $0 \%$ & Valid File \\
\hline Java JSP & $0 \%$ & $100 \%$ & Corrupted File \\
\hline Web HTML & $0 \%$ & $100 \%$ & Corrupted File \\
\hline Image PNG & $100 \%$ & $0 \%$ & Valid File \\
\hline
\end{tabular}

\section{CONCLUSIONS}

We proposed a system for believable URIs to construct advanced artifacts which is provable on the Web, permanent and unique. In the event that increased the significance of sharing or publishing, it could considerably affect the 
process in the Web, which will enhance proficiency, dependability and reliability of instruments utilizing the Web assets, and leads into a critical specialized column for the Semantic Web, specifically for advanced science, where provenance and obviousness are essential.

Investigative information examinations for instance, may be led later on in a completely reproducible way inside information similar to today's product ventures. Likewise, we are dealing with the idea of securing the Nanodistribution files that vulnerable to attacks by the definition and ID of little of Nano productions. Such files are Nano productions themselves and, obviously, are recognized from thisbelievable URIs. The methodology displayed here may significantly contribute to shape the eventual fate of publishing on the Web.

\section{ACKNOWLEDGMENT}

Proposed work is a job of great enormity and it can't be accomplished by an individual all by them. Eventually, I am very much grateful to the Vice Principal and Head of the Department Dr. R.J. Anandhi for her unfailing encouragement and suggestion given to me in the course of my project work.I convey my sincere gratitude to my guide Mrs. Gousia Thaniyath, Assistant professor, Department of CSE, for having constantly guided and monitored the development of the project work.

A note of thanks to the Department of Computer Science Engineering, both teaching and non-teaching staff for their co-operation extended to us.I thank my parents for their constant support and encouragement. Last, but not the least, I would like to thank my peers for the unconditional support.

\section{REFERENCES}

[1] T. Kuhn and M. Dumontier, "Making Digital Artifacts on the Web Verifiable and Reliable," IEEE transaction on knowledge and data engineering Vol no 99 year 2015.

[2] T. Kuhn and M. Dumontier, "Trusty URIs: Verifiable, immutable, and permanent digital artifacts for linked data," in Proceedings of the 11th Extended Semantic Web Conference (ESWC 2014), ser.Lecture Notes in Computer Science. Springer, 2014.

[3] P. Groth, A. Gibson, and J. Velterop, "The anatomy of a nanopublication,"Information Services and Use, vol. 30, no. 1, pp. 51-56,2010.

[4] R. Hoekstra, "The MetaLex document server," in The Semantic Web ISWC 2011. Springer, 2011, pp. 128143.

[5] R. Gentleman, "Reproducible research: A bioinformatics casestudy," Statistical applications in genetics and molecular biology, vol. 4, no. 1, 2005.

[6] R. D. Peng, "Reproducible research in computational science,"Science, vol. 334, no. 6060, p. 1226, 2011.

[7] O. S. Collaboration et al., "An open, large-scale, collaborative effort to estimate the reproducibility of psychological science,"Perspectives on Psychological Science, vol. 7, no. 6, pp. 657-660, 2012.

[8] S. Bechhofer, D. De Roure, M. Gamble, C. Goble, and I. Buchan,"Research objects: Towards exchange and reuse of digital knowledge,"The Future of the Web for Collaborative Science, 2010.

[9] "Secure hash standard (SHS)," National Institute of Standards and Technology (NIST), Tech. Rep. FIPS PUB 180-4, March2012. http://csrc.nist.gov/publications/fips/fips180-4/fips180-4.pdf.

[10] T. Kuhn, C. Chichester, M. Dumontier, and M. Krauthammer, "Publishing without publishers: a decentralized approach to dissemination, retrieval, and archiving of data," arXiv preprint arXiv:1411.2749, 2014. 